\title{
Procédures permettant aux personnes malentendantes de percevoir les avertisseurs sonores en milieu industriel
}

Une nouvelle méthode basée sur une modélisation de la détection auditive permet de prédire la capacité des personnes malentendantes à détecter un signal de fréquence quelconque dans un bruit. Les données normatives de sélectivité fréquentielle permettent d'élargir son champ d'application à des fins de diagnostic. La capacité à percevoir des avertisseurs sonores en milieu de travail ne devrait donc plus présenter un obstacle important à l'embauche des personnes présentant des déficiences auditives.

\section{Raymond Hétu}

\section{Introduction}

Les travaux du Groupe d'acoustique de l'université de Montréal (GAUM) ayant trait aux capacités auditives requises par les milieux de travail ont pour origine, dans une certaine mesure, des demandes de consultation au sujet de cas de refus d'embauche ou de mutations motivés par des incapacités auditives. L'analyse des critères invoqués dans de tels cas a été révélatrices de barrières importantes à l'intégration des personnes présentant des déficiences auditives. L'une des principales raisons justifiant un refus d'emploi en milieu industriel à des personnes malentendantes est l'incapacité à entendre et à reconnaître les avertisseurs sonores de danger. Nous avons entrepris d'examiner en profondeur cette question et de rechercher des solutions qui favoriseraient l'intégration des personnes malentendantes.
Les avertisseurs sonores en industrie

Dans les milieux industriels en général, on s'est fort peu préoccupé de l'audibilité des avertisseurs sonores malgré la présence de bruits ambiants intenses, de réverbération sonore importante et de l'usage de protecteurs anti-bruit. En fait, pratiquement aucun contrôle réglementaire n'est exercé sur l'usage des avertisseurs sonores en milieu industriel [7].

Leur utilisation, pourtant très fréquente dans ces milieux [6], peut constituer une situation de handicap importante pour les personnes qui présentent des déficiences auditives. De plus, parmi les travailleurs expérimentés, les nombreuses victimes de surdité professionnelle vivent vraisemblablement des situations de vulnérabilité à cause du manque d'ajustement des avertisseurs sonores en milieu industriel aux conditions 
ambiantes et aux limites imposées par ce type de surdité [7].

La première solution qui s'impose de façon évidente est la réduction du bruit de manière à permettre à chacun de détecter facilement des signaux sonores d'urgence (et, aussi, de pouvoir utiliser la voix pour communiquer efficacement en situation d'urgence). Compte tenu de la lenteur du processus d'amélioration des ambiances sonores en milieu industriel [2], la détection des avertisseurs sonores dans le bruit pose un sérieux problème aux personnes présentant des déficiences auditives.

\section{Caractérisation}

\section{de la capacité résiduelle}

Dans une perspective d'adaptation de l'environnement de travail aux contraintes imposées par la surdité, le GAUM a entrepris de développer une solution à ce problème. Nous avons d'abord identifié une méthode de mesure de la sélectivité fréquentielle qui permet la prédiction de la capacité de détection sonore dans le bruit [9]. La figure 1 illustre le principe de cette mesure. La capacité de détecter un signal de fréquence quelconque dans un bruit est décrite en termes de filtres auditifs de largeur fréquentielle déterminée. Le filtre

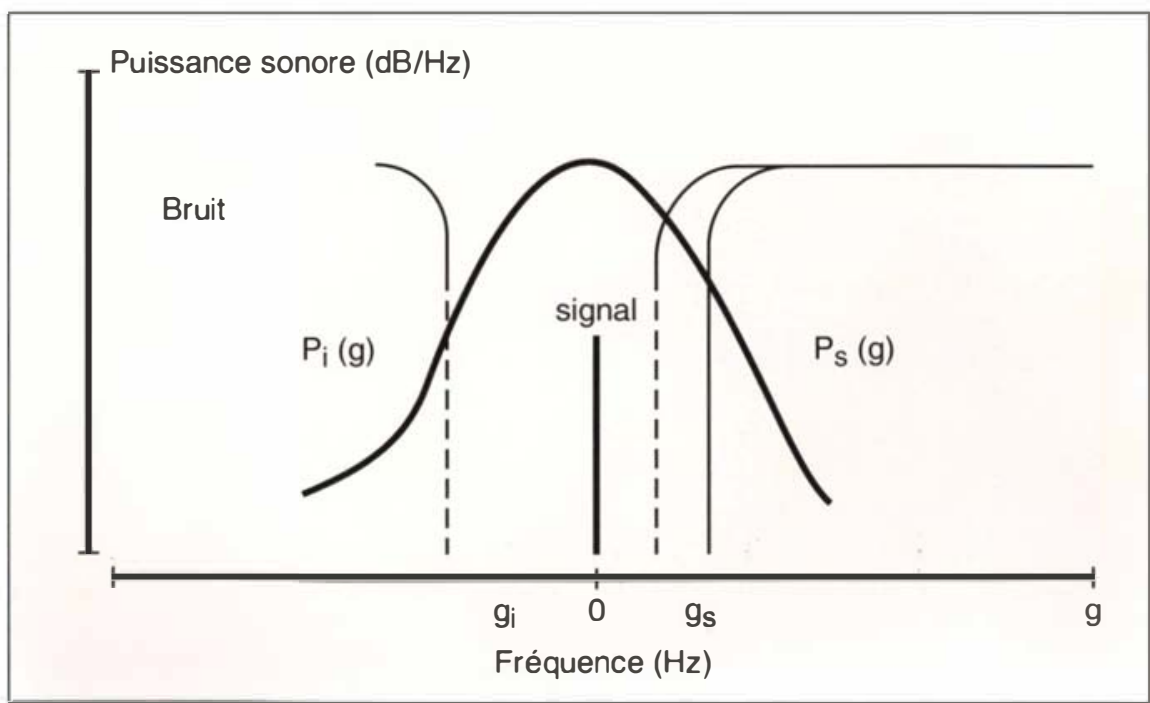

Figure 1. Représentation d'un signal tonal dans l'échancrure de largeur variable (définie d'après les paramètres gi et gs), pratiquée dans le spectre d'un bruit de masque. Les branches supérieures $\left(P_{s}\right)$ et inférieures $\left(P_{1}\right)$ du filtre auditif sont calculées d'après les seuils d'audibilité du signal en présence de bruits de différentes largeurs d'échancrure spectrale.

$\mathrm{m} / \mathrm{s} n^{\circ} 5 \mathrm{vol}$. 9, mai 93 rejette une certaine quantité d'energie sonore associée au bruit ambiant de part et d'autre d'une composante fréquentielle du signal. Ce rejet est d'autant plus important que les pentes du filtre sont raides et que l'on s'éloigne de sa fréquence centre. Pour le caractériser, on détermine le seuil d'audibilité d'un son pur (signal composé d'une seule fréquence) en présence d'un bruit masquant dans le spectre duquel est pratiquée une échancrure dont on varie la largeur (figure 1). Le seuil de détection du signal est défini comme étant directement proportionnel à la puissance du bruit masquant qui passe à travers le filtre auditif centré à la fréquence du signal. Par conséquent, plus étroite est l'échancrure, plus grande sera la quantité de bruit traversant le filtre auditif et inversement.

Dans l'exemple d'un filtre normal représenté à la figure $2 A$, le filtre centré à $1 \mathrm{kHz}$ agit comme un atténuateur pouvant diminuer, à $1,4 \mathrm{kHz}$ par exemple, de $60 \mathrm{~dB}$ le pouvoir masquant du bruit ambiant. La perte de résolution fréquentielle, illustrée à la figure $2 B$, se traduit par un élargissement du filtre auditif à certaines fréquences, accompagné d'un adoucissement plus ou moins important de ses pentes. L'atténuation du bruit masquant est moins forte, et le pouvoir de détection d'un signal dans cette zone de fréquences, beaucoup plus faible. Cette situation exige donc que le signal soit plus intense pour être détecté.

L'intérêt de cette approche réside dans le fait qu'elle permet, moyennant une modélisation de la détection auditive, de prédire la capacité d'un individu donné à détecter un signal quelconque en présence d'un bruit quelconque. A terme, il est possible de définir les caractéristiques d'un signal pour qu'il soit entendu et reconnu par une personne malentendante, en dépit du bruit ambiant.

La mesure des filtres auditifs a été développée dans un laboratoire britannique il $y$ a une quinzaine d'années [8]. Nous avons entrepris d'adapter cette méthode aux contraintes de l'examen clinique. Une étude de faisabilité auprès de travailleurs atteints de surdité professionnelle a confirmé qu'elle était applicable à des personnes ayant différents degrés de perte de sensibilité auditive [5]. Un travail d'optimisation de la procédure a ensuite été réalisé [3]. Ainsi, en moins de 25 minutes, il est possible de caractériser la capacité de détection dans le bruit d'une personne avec un degré satisfaisant de précision. Nous avons, en outre, recueilli des données normatives de sélectivité fréquentielle avec cette méthode de manière à élargir son champ d'application à des fins de diagnostic.

\section{Adaptation de l'environnement}

Un logiciel (Détectson ${ }^{\mathrm{mc}}$ ) avait déjà été mis au point par le GAUM pour prédire, sur une base statistique, les conditions de détection et de reconnaissance des avertisseurs sonores en fonction de l'environnement sonore $[7,10]$. Le logiciel est en voie de modification afin de permettre de spécifier les caractéristiques des signaux sonores qui seraient compatibles avec les capacités individuelles telles qu'évaluées au moyen de l'examen clinique. Nous en sommes actuellement à valider de telles prédictions auprès de personnes présentant divers types et degrés de perte de sensibilité auditive. Au terme de ce travail, nous devrions être en 
mesure de proposer une stratégie de réadaptation professionnelle pour les nombreuses personnes ayant une déficience auditive du fait de leur travail ainsi que pour les personnes atteintes de surdité d'origines diverses, dont le milieu de travail exigerait qu'elles entendent des avertisseurs sonores en ambiances plus ou moins bruyantes.

\section{L'aide auditive en milieu de travail industriel}

Le GAUM a également étudié les conditions de détection d'avertisseurs sonores lorsque l'audition est corrigée au moyen d'une aide auditive $[1,4]$. Les résultats de nos travaux ont montré qu'il était possible de réunir des conditions optimales de détection sonore avec une aide auditive, moyennant certaines conditions techniques. En effet, l'embout de l'aide auditive peut agir comme un atténuateur efficace contre le bruit ambiant si, d'une part, l'empreinte prise pour le fabriquer est parfaite et si, d'autre part, l'embout n'est pas muni d'un évent, c'est-à-dire d'une ouverture permettant la circulation de l'air dans le conduit auditif externe.

On remarque à la figure 3 que l'affaiblissement sonore des embouts est relativement important et ce, quel que soit le modèle d'aide auditive utilisé. Ainsi, à $500 \mathrm{~Hz}$ et au-delà, l'affaiblissement sonore est supérieur à $25 \mathrm{~dB}$. L'embout, s'il est parfaitement étanche, offre donc une barrière efficace au bruit ambiant ; il est alors possible de transmettre à l'aide auditive des signaux sonores via un système émetteur-récepteur FM. Le signal peut être capté par l'aide auditive au moyen de sa bobine d'induction ou d'un couplage électronique direct pour ensuite être amplifié selon les besoins de la personne malentendante. Étant donné l'affaiblissement sonore du bruit ambiant par l'embout, d'une part, et l'amplification du signal par l'aide auditive, d'autre part, le problème de détection sonore en milieu plus ou moins bruyant et réverbérant peut très bien être résolu même si la perte d'audition de la personne est relativement importante. Le même dispositif est évidemment utilisable pour la transmission efficace des messages verbaux.

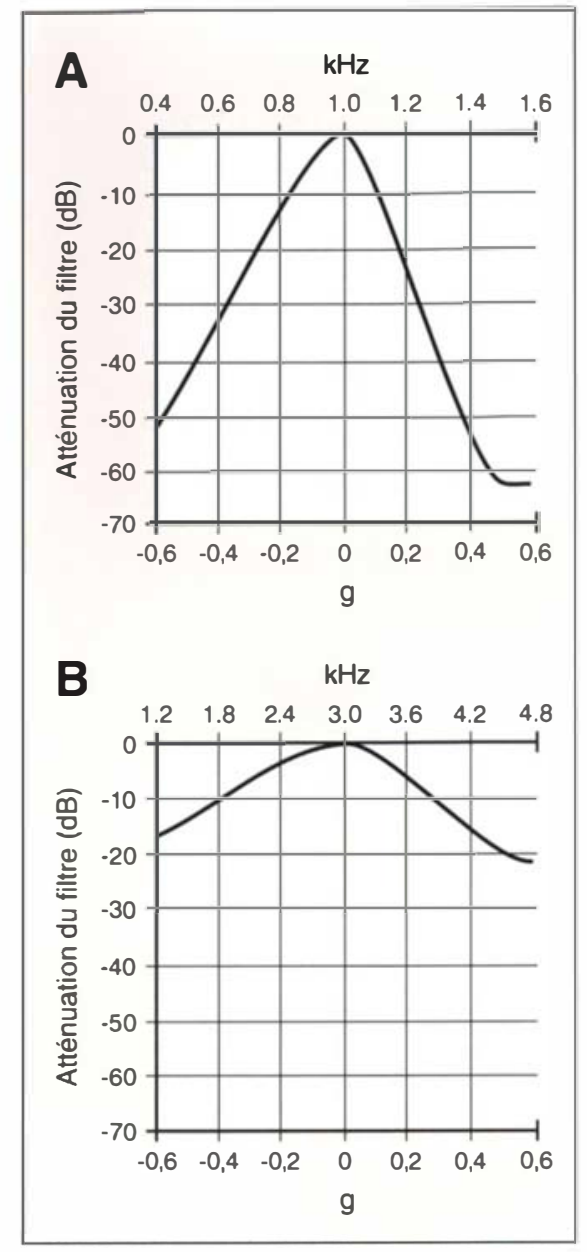

Figure 2. Exemple de filtres auditifs centrés à $1000 \mathrm{~Hz}$ (A) et à $3000 \mathrm{~Hz}$ (B), mesurés à l'oreille droite d'un travailleur exposé au bruit, au moyen de la technique du bruit à échancrure. Les seuils absolus d'audition étaient de 10 et de $43 \mathrm{~dB} H \mathrm{HL}$ à 1000 et à $3000 \mathrm{~Hz}$ respectivement.

\section{Conclusion}

Nous disposons dorénavant de moyens techniques relativement simples pour permettre d'adapter les caractéristiques des signaux sonores aux contraintes imposées par des déficiences auditives, en intervenant sur leurs sources ou encore sur leur mode de transmission et de réception. Le risque d'accident associé à une baisse de capacité à percevoir des avertisseurs sonores en milieu de travail ne devrait donc plus constituer une barrière à l'embauche des personnes malentendantes normals and hearing impaired individuals. In : Mattila M. and Karwowski W, eds. 1992 : 105-12. 


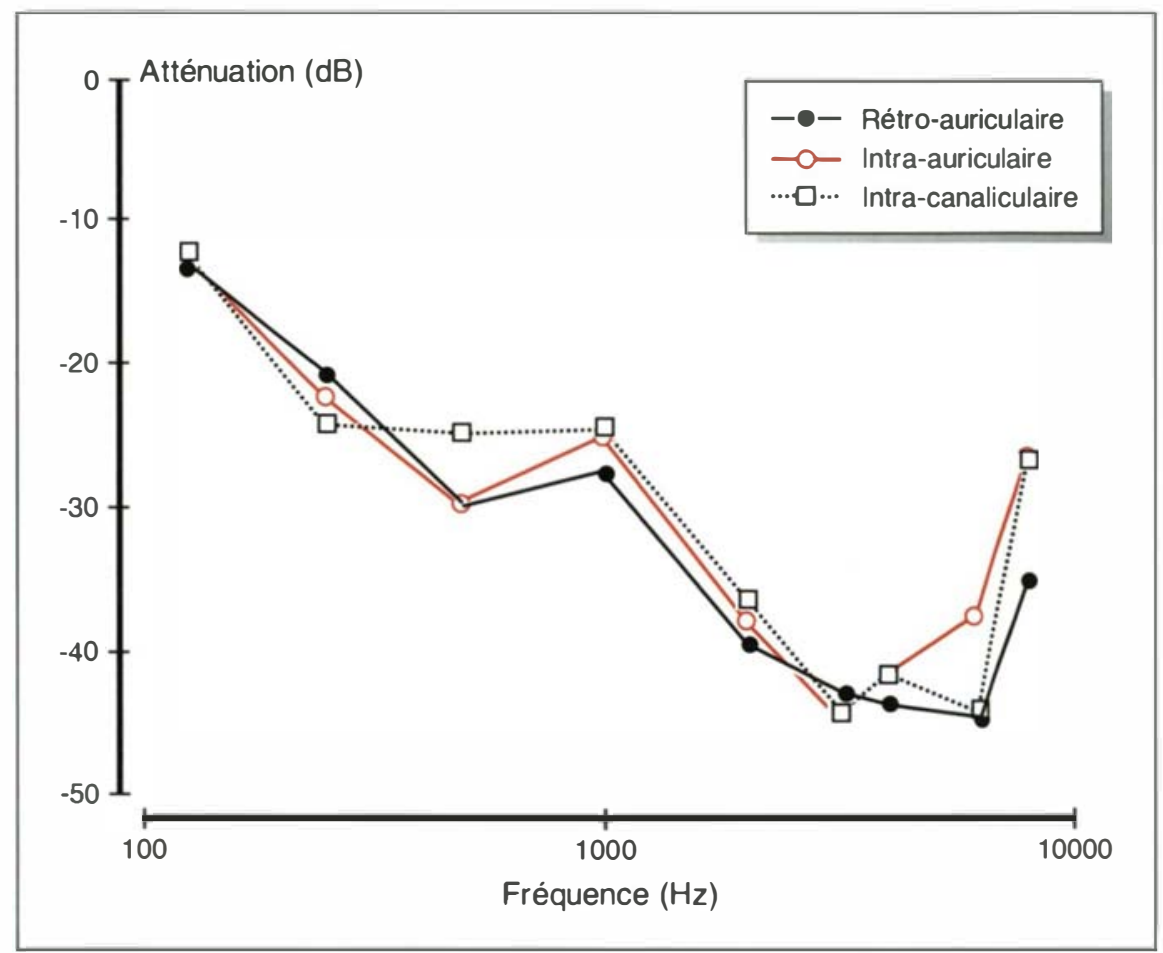

Figure 3. Affaiblissement sonore moyen offert par les embouts de trois modèles d'aides auditives (rétro-auriculaire, intra-auriculaire et intracanaliculairel, lorsqu'ils sont parfaitement étanches. Les valeurs sont obtenues à partir de corrections des pertes par insertion mesurées sur un modèle mécano-acoustique de la tête et de l'oreille humaine [1].

\section{Summary}

Procedures allowing hearing impaired workers to perceive sound warning signals

The inability of hearing impaired individuals to perceive acoustic warning signals has been used to refuse them jobs in industry. The Groupe d'acoustique de l'université de Montréal has undertaken a research program to find means to raise this barrier to the integration of people with hearing impairments. A clinical method was devised to assess individual capabilities of signal detection in noise. Using a computer model of auditory detection developed in our laboratory, it is now possible to specify the signal features that can make them perceivable by individuals with hearing impairments. A project has also been undertaken to determine adequate conditions of use of hearing aids in industry. Our results showed that it is possible to take advantage of the earmold to act as a hearing protector, and of the amplifier to adjust signals, sent by FM transmitters, to levels that are easily perceivable. According to these findings, the risk of accidents associated with a reduced capacity to perceive sound warning signals should not constitue a barrier anymore for the integration of people with hearing impairments in industrial workplaces. 